\title{
Reduction of acetaminophen interference in glucose sensors by a composite Nafion membrane: demonstration in rats and man
}

\author{
D.Moatti-Sirat ${ }^{1}$, V.Poitout ${ }^{1}$, V.Thomé ${ }^{1}$, M. N. Gangnerau ${ }^{1}$, Y.Zhang ${ }^{2}$, Y.Hu ${ }^{2}$, G. S. Wilson ${ }^{2}$, F. Lemonnier ${ }^{3}$, \\ J.C.Klein ${ }^{3}$, G. Reach ${ }^{1}$ \\ ${ }^{1}$ INSERM U341, Service de Diabétologie, Hôtel-Dieu, Paris, France \\ ${ }^{2}$ Department of Chemistry, University of Kansas, Lawrence, Kansas, USA \\ ${ }^{3}$ Centre de Morphologie Mathématique, Ecole des Mines de Paris, Fontainebleau, France
}

\begin{abstract}
Summary Amperometric glucose sensors typically monitor the production of hydrogen peroxide generated in the course of the enzymatic oxidation of glucose. At the applied potential necessary to oxidize the peroxide produced, other species are also electroactive and contribute to the signal. Interference of ascorbate or urate has been effectively eliminated, but that resulting from the widely used analgesic acetaminophen is not. The aim of this work was to reduce this interference, which was found to be possible by introducing a membrane constructed of Nafion. We compared the in vitro sensitivity to acetaminophen of five Nafion sensors with that of five non-Nafion sensors with identical glucose sensitivity $\left(2.0 \pm 0.4\right.$ vs $1.9 \pm 0.1 \mathrm{nA} \cdot \mathrm{mmol}^{-1}$. $\left.1^{-1}, \mathrm{NS}\right)$ : sensitivity to acetaminophen was $12.2 \pm 2.7 \mathrm{vs}$ $30.8 \pm 6.3 \mathrm{nA} \cdot \mathrm{mmol}^{-1} \cdot 1^{-1}$, respectively $(p<0.05)$. These sensors were tested in rats by implanting in each animal one Nafion and one non-Nafion sensors. The in vivo sensitivity to glucose was similar $(0.33 \pm 0.09$ vs $0.30 \pm 0.05 \mathrm{nA} \cdot \mathrm{mmol}^{-1} \cdot 1^{-1}$, NS). The current gener-
\end{abstract}

ated by an acetaminophen infusion (plasma acetaminophen plateau $=140 \pm 10 \mu \mathrm{mol} / 1$ ) was much decreased in the case of the Nafion sensor: $0.5 \pm 0.3$ vs $2.0 \pm 0.7 \mathrm{nA}, p<0.05$ ). Five Nafion sensors were implanted in the subcutaneous tissue of normal human volunteers who were given on oral dose of $500 \mathrm{mg}$ acetaminophen. No change in the sensor current was observed, although plasma acetaminophen reached a peak $(35 \pm 6 \mu \mathrm{mol} / \mathrm{l})$ at $60 \mathrm{~min}$ and decreased by $50 \%$ at $180 \mathrm{~min}$. In contrast, the current increased from $2.1 \pm 0.7$ to $3.9 \pm 1.2 \mathrm{nA}(p<0.05)$ at $60 \mathrm{~min}$ of a subsequent oral glucose tolerance test when plasma glucose concentration increased from $4.8 \pm 0.3$ to $8.0 \pm 0.9 \mathrm{mmol} / \mathrm{l}$. This paper provides the evidence that the presence of a Nafion membrane is a solution to acetaminophen interference in glucose sensing. [Diabetologia (1994) 37:610-616]

Key words Glucose sensor, interference, acetaminophen, Nafion.
The specificity of the response represents a basic requirement in the development of a glucose sensor. In most of the sensors developed so far, enzymatic recognition of glucose is used to provide this specificity: glucose oxidation by glucose oxidase produces gluconic acid and hydrogen peroxide. A second step of the reac-

Received: 2 September 1993

and in revised form: 2 January 1994

Corresponding author: Dr. G. Reach, INSERM U341, Service de Diabétologie, Hôtel-Dieu, 1, Place du Parvis Notre Dame, F75004 Paris, France

Abbreviations: OGTT, Oral glucose tolerance test tion is the oxidation of hydrogen peroxide, generating electrons recorded as a current. This step requires the presence of an applied potential of about $600 \mathrm{mV}$ at the working electrode. Any substance with access to the electrode and electroactive at this potential will therefore generate an interfering current, causing an error in the estimation of the glucose concentration. Several endogenous analytes, such as ascorbate or urate, or exogenous drug such as acetaminophen, are known to be potentially interfering agents [1]. Their interfering effects have been investigated under in vitro conditions [2-4] and for some of them under in vivo conditions [4$6]$. It has been recognized that cellulose acetate membranes, typically present in the sensor, efficiently eliminate interference by urate and ascorbate $[3,7,8]$. How- 
ever, this is not the case of acetaminophen, and the interfering effect of this most commonly used analgesic cannot be overlooked.

One approach to minimization of this interfering effect is the incorporation of an additional membrane, with pore size and chemical selectivity that could specifically retard diffusion of the interferent. Our laboratories have tested several different membranes, and after preliminary results have chosen to include a composite Nafion membrane (Zhang, unpublished data). We present herein the demonstration of the effect of such a Nafion membrane on acetaminophen interference, as assessed in vitro, and in vivo in rats and in human volunteers.

\section{Materials and methods}

\section{The needle-type glucose sensor}

The sensor consists of a platinum-iridium wire coated with teflon ( $0.25 \mathrm{~mm}$ outer diameter) except for a $1.5 \mathrm{~mm}$ cavity near the extremity where membranes were deposited: an enzymatic layer (glucose oxidase immobilized on cellulose acetate, reticulated with glutaraldehyde), a polyurethane layer. Details of the sensor's design have been published elsewhere [7]. The additional composite Nafion membrane is prepared by the alternative deposition of cellulose acetate and Nafion solutions on the bare part of the sensor cavity. Nafion ( $5 \%$ solution in lower aliphatic alcohols and $10 \%$ water, Aldrich, Milwaukee, Wis., USA) is a polymer of perfluorosulfonic acid that has a Teflon-like structure. When combined with cellulose acetate it forms a composite membrane which probably has a segregated structure with polar and non-polar domains. The cellulose acetate is deposited by dipping the sensor in a $4.5 \%$ acetone/ethanol $(2: 1 \mathrm{v}: \mathrm{v})$ solution of cellulose acetate followed by drying at room temperature for $10 \mathrm{~min}$. This step is then repeated. A three-turn wire loop (diameter $3 \mathrm{~mm}$ ) is constructed from $0.7 \mathrm{~mm}$ outer diameter copper wire. This loop was necessary to deliver a precisely known volume of low viscosity Nafion solution to the cavity surface. The inside of the loop is filled with the Nafion solution. The cavity of the sensor is then passed through the loop horizontally first in one direction and then the other. The sensor is dried at room temperature for $10 \mathrm{~min}$ and the procedure is repeated two more times. The above cycle of cellulose acetate and Nafion deposition is repeated two more times, before enzyme and polyurethane deposition.

\section{In vitro study}

In a first set of experiments, in which the sensors were evaluated both in vitro and in vivo in rats, ten sensors were evaluated under in vitro conditions immediately before implantation. In order to assess the efficiency of the Nafion layer in limiting the acetaminophen access to the anode, two kinds of sensors, constructed either with, or without Nafion were compared. The in vitro characteristics of the sensors were determined in a stirred, buffered solution $\left(0.15 \mathrm{~mol} / /\right.$ phosphate, $\left.0.15 \mathrm{~mol} / 1 \mathrm{NaCl}, \mathrm{pH} 7.4,37^{\circ} \mathrm{C}\right)$. The sensors were connected to an amperometric unit (PRG-Del; Solea Tacussel Electronique, Villeurbanne, France) applying a $590 \mathrm{mV}$ potential between the anode and the cathode. The signal was recorded with a chart-recorder (SE 120; Goerz Electro,
Vienna, Austria). In vitro responses to glucose (concentration ranging from 0 to $20 \mathrm{mmol} / \mathrm{l}$ ) and acetaminophen (concentration from 0 to $200 \mu \mathrm{mol} / \mathrm{l}$ ) were determined in phosphate buffer. The sensitivity to glucose and acetaminophen $(\Delta \mathrm{l} / \Delta \mathrm{C}$ expressed in $\left.\mathrm{nA} \cdot \mathrm{mmol}^{-1} \cdot 1^{-1}\right)$ and the response time to glucose and acetaminophen (time to reach $90 \%$ of the response, expressed in min), were compared for the Nafion and non-Nafion sensors. The acetaminophen concentration was chosen to simulate the plasma concentration in human subjects observed after maximum therapeutic intake: 100 to $200 \mu \mathrm{mol} / 130 \mathrm{~min}$ after $1 \mathrm{~g}$ intake $[9,10]$.

\section{In vivo trials in rats}

Overnight-fasted normal Wistar rats (250-300 g body weight) were anaesthetized with halothane. The animals were warmed under a lamp to prevent vasoconstriction. Polyethylene and silicone catheters were inserted into the left jugular vein and the contralateral carotid artery, for glucose or acetaminophen infusion and blood sampling, respectively. One Nafion and one nonNafion sensor were then inserted through a 20 gauge cannula, in the subscapular subcutaneous tissue. The cannula was then removed, leaving the sensors in place, which were then connected to the amperometric units. A run-in period was required to obtain stable currents, the test beginning when the two sensors' currents were stable (time 0 ).

In the first part of the experiment, glucose was infused over $40 \mathrm{~min}$ at a rate of 10 to $15 \mathrm{mg} \cdot \mathrm{kg}^{-1} \cdot \mathrm{min}^{-1}$ to reach a plateau over 5 to 15 min. Glucose infusion was then interrupted so that glycaemia and currents returned to previous levels. Stabilizations were obtained from 120 to $170 \mathrm{~min}$. Acetaminophen, in the form of propacetamol chlorhydrate (Prodafalgan; UPSA, Agen, France) was then infused: a priming dose of $40 \mu \mathrm{mol}$ was followed by a constant infusion of $2.7 \mu \mathrm{mol} \cdot \mathrm{kg}^{-1} \cdot \mathrm{min}^{-1}$ over $1 \mathrm{~h}$ to attain a plasma acetaminophen level of $140 \pm 10 \mu \mathrm{mol} / \mathrm{l}$. Plasma glucose concentration was determined in arterial blood samples at 5-min intervals during the first $120 \mathrm{~min}$ (10 corresponding to the beginning of glucose infusion). Immediately before, and during the acetaminophen infusion $(5,10,15,30$ and $60 \mathrm{~min}$ after initiation), 1-ml blood samples were drawn, to determine both glucose and acetaminophen concentrations. Samples were immediately centrifuged, plasma separated for analysis, the cell pellet re-suspended with saline and immediately re-injected into the rat to replace the lost volume of blood.

In order to transform the recorded current into an estimate of the subcutaneous glucose concentration, a two point calibration procedure was used as previously described [11], providing an in vivo sensitivity to glucose, S, and the current extrapolated in the absence of glucose, Io. The determination of these parameters took into account two sets of values of the current and the glycaemia, obtained immediately before and at the end of the glucose infusion. The estimate of the subcutaneous glucose concentration $\mathrm{SCG}(\mathrm{t})$ was derived at any time from the current and the in vivo parameters, according to the formula: $\operatorname{SCG}(\mathrm{t})=(\mathrm{I}(\mathrm{t})-\mathrm{Io}) / \mathrm{S}$.

\section{Evaluation in human volunteers}

The glucose monitoring system. Glucose sensors used in the human trials were identical to the Nafion sensors evaluated in rats. They were sterilized by exposure to ethylene oxide, (supplied by Kansas University) in a sterile package and unpacked at the time of the implantation, several weeks after sterilization.

In these trials, the monitoring unit consisted of a $6 \times 12 \times 18 \mathrm{~cm}$, battery-driven, electronic device designed to 
Table 1. In vitro and in vivo characteristics of the Nafion and non-Nafion glucose sensors implanted in rats. The bias was defined as the glucose concentration which would produce the same current as that generated by $200 \mu$ mol/1 acetaminophen

\begin{tabular}{lllcc}
\hline & Parameters & Analytes & Non-Nafion sensors & Nafion sensors \\
\hline In vitro & $\Delta \mathrm{I} / \Delta \mathrm{C}$ & Glucose & $1.9 \pm 0.1$ & $2.0 \pm 0.4$ \\
& $\left(\mathrm{nA} \cdot \mathrm{mmol}^{-1} \cdot \mathrm{I}^{-1}\right)$ & Acetaminophen & $30.8 \pm 6.3$ & $12.2 \pm 2.7$ \\
& Io $(\mathrm{nA})$ & & $1.8 \pm 0.5$ & $1.9 \pm 0.5$ \\
& Bias & & $3.4 \pm 0.8$ & $1.4 \pm 0.3$ \\
& $(\mathrm{mmol} /$ glucose) & & $2.6 \pm 0.2$ & $3.6 \pm 1.0$ \\
& Response time & Glucose & $32 \pm 6$ & $42 \pm 7$ \\
In vivo & Acetaminophen & $0.30 \pm 0.05$ & $0.33 \pm 0.09$ \\
& $\begin{array}{l}\mathrm{min} / \Delta \mathrm{C} \\
\left(\mathrm{nA} \cdot \mathrm{mmol}^{-1} \cdot \mathrm{1}^{-1}\right)\end{array}$ & Glucose & $15.3 \pm 1.2$ & $3.5 \pm 1.1$ \\
& Io & & & $0.8 \pm 0.5$ \\
& $(\mathrm{nA})$ & & $1.0 \pm 0.1$ & \\
& Bias & & $11.7 \pm 2.4$ & $2.8 \pm 1.0$ \\
\hline
\end{tabular}

Values shown are mean $\pm \mathrm{SEM}, n=5$ for both groups

apply a $590 \mathrm{mV}$ potential between the electrodes, to measure, to filter, and to display the current. This device was designed and produced by the Centre de Morphologie Mathématique, Ecole des Mines de Paris, Fontainebleau, in the framework of this research programme. It has a memory which allows the storage of up to 8000 current values. It can be connected to a portable PC (Toshiba T1200 XE; Toshiba Corp., Tokyo, Japan) to upload the stored values for data postprocessing. This system, consisting of a battery-driven electronic control unit connected for the purpose of calibration to a portable $\mathrm{PC}$, was never connected to the main. The potential, the time between two measurements and the filtering characteristics can be chosen by the operator, using specially designed software. Moreover, the unit is able to display the estimation of the glucose concentration calculated from the current in real time (described later in this paper).

Subjects. Five non-diabetic human volunteers (four women and one man) participated in this study. All subjects gave their informed consent, and the study was approved by the Ethical Committee of Hôtel-Dieu Hospital (Comité Consultatif pour la Protection des Personnes qui se prêtent à la Recherche Biomédicale, according to the Huriet-Serusclat law). The age of the subjects was $26.0 \pm 0.5$ years (mean $\pm \mathrm{SD}$, range $25-28$ years). The $\mathrm{BMI}$ was $21.6 \pm 0.7 \mathrm{~kg} / \mathrm{m}^{2}$ (mean $\pm \mathrm{SD}$, range $20.6-24.3$ ).

Sensor implantation. At 18.00 hours, a 21 gauge needle was inserted over a distance of $1 \mathrm{~cm}$ into the subcutaneous tissue of the outer face of the forearm. The sensor was indwelled through the needle which was then pulled out, leaving the sensor in place. No local anaesthesia was used. The sensor was connected to a small battery applying a $590 \mathrm{mV}$ potential. After the subject fasted overnight, the sensor was connected at 08.00 hours to the monitoring unit. The current was measured and recorded every $30 \mathrm{~s}$. The unit was programmed to display the median of the five last values of current. The signal was then allowed to stabilize for a 2-h period.

Acetaminophen administration, OGTT and calibration. A 20 gauge catheter was indwelled in a forearm vein for blood sampling. At t0, a $500 \mathrm{mg}$ acetaminophen tablet was given orally. At $\mathrm{t} 0=60 \mathrm{~min}$, a solution of $75 \mathrm{~g}$ of glucose in $135 \mathrm{ml}$ of water was ingested by the subjects. Plasma acetaminophen was measured at $0,15,30,45,60 \mathrm{~min}$ and in one subject at 120, 180 and $240 \mathrm{~min}$. Plasma glucose was measured every $15 \mathrm{~min}$ over a $4 \mathrm{~h}$ period. The basal value of blood glucose $(\mathrm{G} 1)$ and its time of measurement (t1) were recorded. About 60 min (time t2) after the ingestion of the glucose, the current reached a stable plateau corresponding to the hyperglycaemic blood glucose value, G2. $\mathrm{G} 1, \mathrm{G} 2, \mathrm{t} 1$ and $\mathrm{t} 2$ were loaded into the monitoring unit. The software then searched for the currents corresponding to $\mathrm{t} 1$ and $\mathrm{t} 2$ $[11,12]$ in the memory of the unit. Sensor calibration was performed by using these two sets of values. The unit then transformed every subsequent measurement of the current into an estimation of the subcutaneous glucose concentration by using the formula given above. A switch on the front of the unit made it possible to display this estimation instead of the current every $30 \mathrm{~s}$. These estimations were noted by the subject over the next $2 \mathrm{~h}$.

The acetaminophen level reached $35 \pm 6 \mu \mathrm{mol} / \mathrm{l}(n=5)$ at $60 \mathrm{~min}$. In one of these subjects, plasma acetaminophen concentration was determined at 120,180 and 240 min and was 36,31 and $19 \mu \mathrm{mol} / \mathrm{l}$, respectively. These values were similar to those observed in three different subjects in whom plasma acetaminophen was determined ( $33 \pm 6 \mu \mathrm{mol} / \mathrm{l}$ at $60 \mathrm{~min}, 25 \pm 2 \mu \mathrm{mol} / \mathrm{l}$ at $120 \mathrm{~min}, 20 \pm 4 \mu \mathrm{mol} / 1$ at $180 \mathrm{~min}$, and $19 \pm 5 \mu \mathrm{mol} / 1 \mathrm{lat} 240 \mathrm{~min}$ ) indicating that between time 60 and $240 \mathrm{~min}$, plasma acetaminophen concentrations decreased by almost a factor two. During the subsequent OGTT, the blood glucose concentration increased from $4.8 \pm 0.3 \mathrm{mmol} / 1$ to $8.0 \pm 0.9 \mathrm{mmol} / \mathrm{l}$ peak value $(n=5)$.

\section{Analytical procedures and data analysis}

Plasma glucose concentration was measured on a Beckman Analyzer (Fullerton, Calif., USA). Plasma acetaminophen concentration was measured with a commercial kit based on fluorescence polarization immunoassay (Reactif Paracetamol Test; Abbot Diagnostic, Rungis, France).

The bias introduced under in vitro conditions by acetaminophen on glucose measurement was defined as the glucose concentration which would produce the same current as that generated by $200 \mu \mathrm{mol} / \mathrm{l}$ acetaminophen.

The magnitude of the bias introduced in vivo in the experiment performed in rats by acetaminophen infusion was determined by comparing the estimate of glucose concentration (calculated on the basis of the two point calibration using changes in the current and in blood glucose concentration which occurred 
at the end of the glucose infusion) with the actual glycaemia determined at the acetaminophen plateau.

The bias introduced by the ingestion of acetaminophen on the subsequent estimation of glucose concentration during the OGTT was determined in the human trials as follows: the plasma glucose concentration at the end of the OGTT (time 240 of the trial) was compared to the estimation of glucose concentration at this time. This estimation was calculated by taking into account the in vivo sensitivity and the background current Io computed from the current and plasma glucose concentration measured at time 60 and 120 min of the trial, i. e. the entire calibration procedure being performed under constantly varying acetaminophen concentration. Furthermore, in order to assess the accuracy of the estimates of glucose concentration, for each single experiment, the estimations of subcutaneous glucose level were compared to the blood glucose concentration from $\mathrm{t}=120 \mathrm{~min}$ (after the calibration) to the end of the test $(\mathrm{t}=240 \mathrm{~min})$.

\section{Statistical analysis}

Results are given as means and SEM: the significance of the data was assessed with the Wilcoxon test, and differences were considered statistically significant for values of $p<0.05$.

\section{Results}

\section{Evaluation in rats}

The in vitro sensitivities to glucose of those sensors evaluated in the experiments performed in rats are shown on Table 1 . No differences were observed between the non-Nafion and Nafion sensors. By contrast, the in vitro sensitivity to acetaminophen was significantly lower for the Nafion sensors $(p<0.05)$. For both Nafion and non-Nafion sensors, the in vitro response time to acetaminophen was consistently longer than that for glucose $(p<0.05)$. Response time to acetaminophen was significantly longer $(p<0.05)$ for the Nafion sensors.

For the non-Nafion sensors, the current generated by $200 \mu \mathrm{mol} / 1$ acetaminophen was $6.2 \pm 1.3 \mathrm{nA}$, which would be the current observed in the presence of $3.4 \pm 0.8 \mathrm{mmol} / \mathrm{l}$ glucose. For the Nafion sensors, the current was $2.5 \pm 0.5 \mathrm{nA}$, which would be the current observed in the presence of $1.4 \pm 0.3 \mathrm{mmol} / 1$ glucose. The reduction in the bias was significant $(p<0.05)$.

Figure 1 shows the results of one in vivo experiment in a rat. The non-Nafion and the Nafion sensors implanted in the same rat had an identical in vitro sensitivity to glucose before implantation $\left(1.7 \mathrm{nA} \cdot \mathrm{mmol}^{-1}\right.$. $\left.\mathrm{I}^{-1}\right)$. The upper panel shows the plasma glucose concentration and acetaminophen concentration during glucose and acetaminophen infusions (the run-in time is not shown on the figures). The middle panel shows the responses of the non-Nafion and the Nafion sensors. The increase of the current due to $135 \mu \mathrm{mol} / \mathrm{l}$ of acetaminophen reached $1.78 \mathrm{nA}$ and $0.42 \mathrm{nA}$ for the non-Nafion and the Nafion sensors, respectively. The
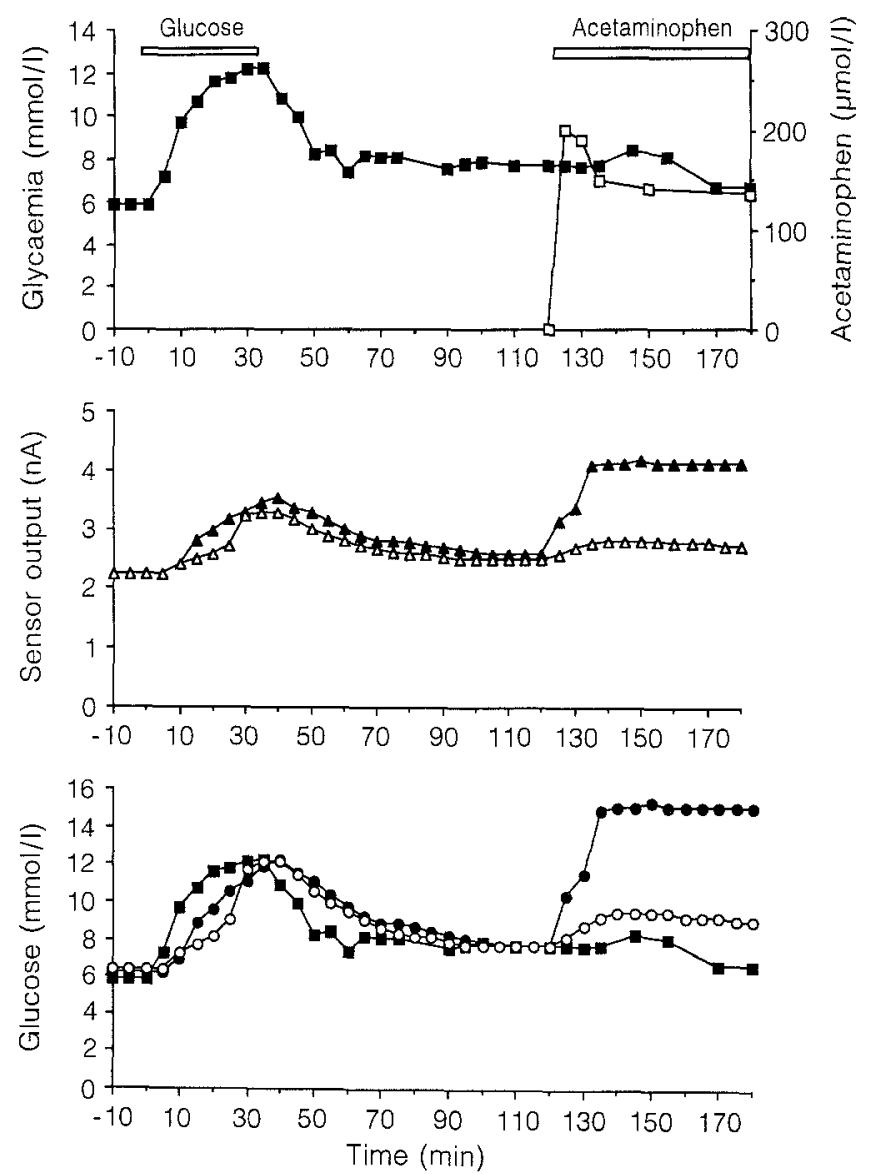

Fig.1. Results of an individual experiment in a rat. The upper panel shows the blood glucose (closed aquares) and acetaminophen (open squares) concentrations. The middle panel represents the current generated by the Nafion (open triangles) and the non-Nafion sensors (closed triangles). The lower curves represent the estimations of the glucose concentrations calculated from the current generated either by the Nafion (open circles) or the non-Nafion (closed-circles) sensors, as compared to the actual blood glucose (closed squares)

lower panel shows the plasma glucose concentration and estimation of the subcutaneous glucose concentration, calculated from the currents and the in vivo parameters. In this experiment, the bias due to these interfering currents reached $8.6 \mathrm{mmol} / 1$ for the non-Nafion sensor and $2.5 \mathrm{mmol} / \mathrm{l}$ for the Nafion sensor.

Summarizing the results observed in five experiments, the time required for signal stabilization was not different for the non-Nafion and the Nafion sensors ( $3 \mathrm{~h} 10 \mathrm{~min} \pm 5 \mathrm{~min}$ and $3 \mathrm{~h} 30 \mathrm{~min} \pm 5 \mathrm{~min}$, respectively, NS). After $40 \pm 10 \mathrm{~min}$ of stable signals, the glucose infusion was performed which increased the glycaemia from $5.3 \pm 0.3$ to $10.3 \pm 0.6 \mathrm{mmol} / 1$ for 5 to $15 \mathrm{~min}$. The current increased at the same time from $2.3 \pm 0.2$ to $3.6 \pm 0.4 \mathrm{nA}$ for the non-Nafion sensors and from $2.5 \pm 0.5$ to $3.6 \pm 0.7 \mathrm{nA}(p<0.05)$ for the Nafion sensors. The magnitude of the increase was not significantly different for the two kinds of sensors. The infusion was then interrupted: the glycaemia decreased to $6.9 \pm 0.2 \mathrm{mmol} / 1$ and the current to $2.7 \pm 0.3 \mathrm{nA}$ and 


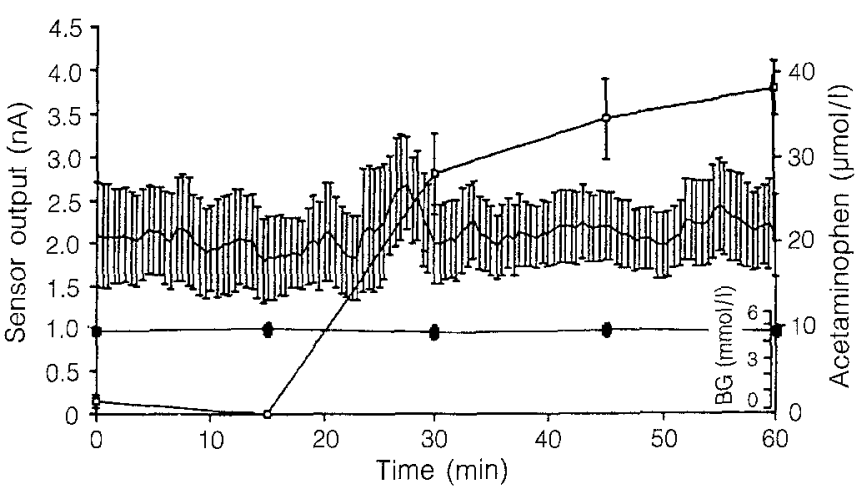

Fig. 2. Results of the five experiments in human volunteers, during the first hour following acetaminophen intake, showing the mean $\pm S E M$ of the blood glucose concentration (BG) during the first hour of the OGTT (closed squares) the mean \pm SEM of the blood acetaminophen concentration following the ingestion of the tablet (open squares), and the mean \pm SEM of the current delivered by the sensors (line)
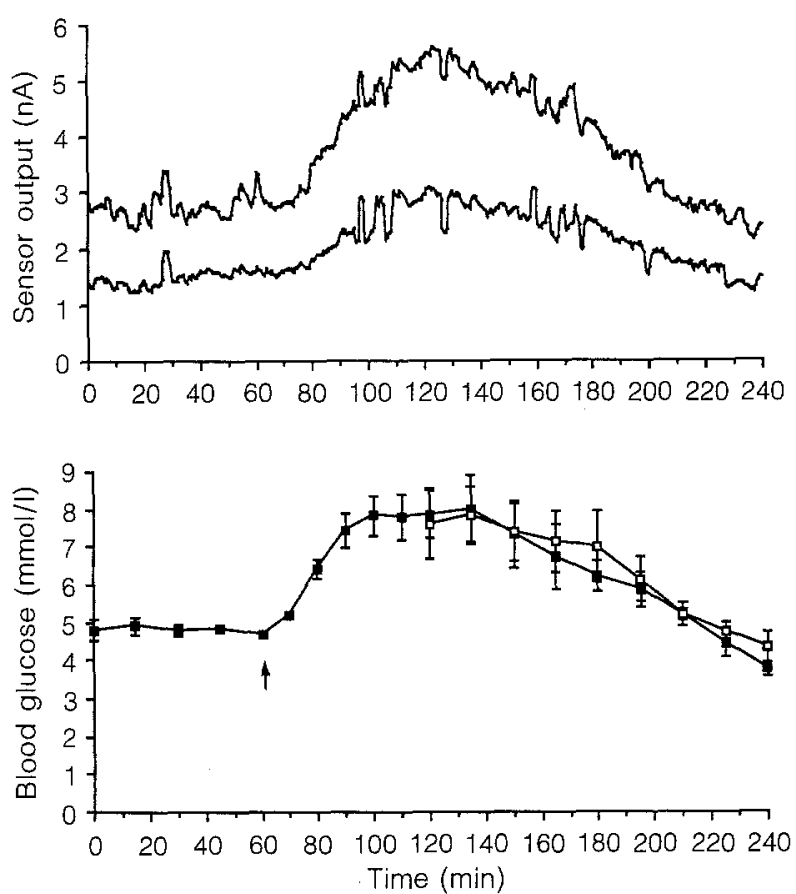

Fig. 3. Results of the five experiments in human volunteers. The upper curve represents the mean \pm SEM and the mean - SEM of the current delivered by the sensors. The lower panel represents the mean \pm SEM of the estimations by the glucose monitoring system of the glucose concentration (open squares), as compared to the blood glucose (closed squares), the arrow indicates the time of glucose ingestion

$2.5 \pm 0.6 \mathrm{nA}(p<0.05)$ for the non-Nafion and the Nafion sensors, respectively. As shown on Table 1, the in vivo sensitivity to glucose, calculated from the increases of glycaemia and current, was similar for the non-Nafion and the Nafion sensors $(0.30 \pm 0.5 \mathrm{nA}$. $\mathrm{mmol}^{-1} \cdot 1^{-1}$ and $0.33 \pm 0.09 \mathrm{nA} \cdot \mathrm{mmol}^{-1} \cdot \mathrm{l}^{-1}$, NS). The magnitude of the reduction in the sensitivity to glucose observed in vivo, as compared to that determined in vitro, was not significantly different for the two kinds of sensors. The acetaminophen infusion caused the acetaminophen concentration to reach a plateau in arterial blood of about $140 \pm 10 \mu \mathrm{mol} / \mathrm{l}$, after a transient increase to a peak of $290 \pm 80 \mu \mathrm{mol} / \mathrm{l}$. The increase in the current due to the acetaminophen concentration was $2.0 \pm 0.7 \mathrm{nA}$ for the non-Nafion sensors and $0.5 \pm 0.3 \mathrm{nA}$ for the Nafion sensors, respectively $(p<0.05)$. The bias introduced by these interfering currents, calculated by subtracting the actual plasma glucose concentration from the glucose concentration estimate, was $9.8 \pm 2.1 \mathrm{mmol} / \mathrm{l}$ for the non-Nafion sensors and $2.4 \pm 0.7 \mathrm{mmol} / \mathrm{l}$ for the Nafion sensors. From the blood acetaminophen concentrations and the increases of current it was possible to estimate an apparent in vivo sensitivity to acetaminophen for the nonNafion and the Nafion sensors: it was significantly lower in the case of the Nafion sensors: $3.7 \pm 1.1 \mathrm{nA}$. $\mathrm{mmol}^{-1} \cdot 1^{-1}$ vs $15.3 \pm 1.2 \mathrm{nA} \cdot \mathrm{mmol}^{-1} \cdot \mathrm{1}^{-1}(p<0.05)$.

\section{Evaluation in human volunteers}

The sensors (Nafion membrane included) used in these trials had an in vitro sensitivity tested before sterilization (i. e. several weeks before the in vivo implantation) of $3.0 \pm 0.4 \mathrm{nA} \cdot \mathrm{mmol}^{-1} \cdot \mathrm{l}^{-1}$, for glucose and $9.8 \pm 2.2 \mathrm{nA} \cdot \mathrm{mmol}^{-1} \cdot 1^{-1}$ for acetaminophen $(n=5)$.

Figure 2 represents the mean blood glucose, acetaminophen concentration and sensor output during the first hour following acetaminophen intake. The current delivered by the sensor remained stable following acetaminophen ingestion (from $2.1 \pm 0.7 \mathrm{nA}$ to $2.4 \pm 0.8 \mathrm{nA}, \mathrm{NS}$ ) despite a significant increase in plasma acetaminophen to $35 \pm 6 \mu \mathrm{mol} / 1$. Following glucose ingestion (Fig. 3), the plasma glucose concentration increased from $4.8 \pm 0.3$ to $8.0 \pm 0.9 \mathrm{mmol} / \mathrm{l}$ at $60 \mathrm{~min}$. The current increased in all cases from $2.1 \pm 0.7$ to $3.9 \pm 1.2 \mathrm{nA}(p<0.05)$, the in vivo sensitivity therefore being $0.60 \pm 0.18 \mathrm{nA} \cdot \mathrm{mmol}^{-1} \cdot 1^{-1}$. The estimation of glucose through the calibration procedure followed the changes in plasma glucose concentration and at 240 min was virtually identical to the concomitant plasma glucose concentration ( $4.0 \pm 0.4$ vs $3.8 \pm 0.2 \mathrm{mmol} / \mathrm{l}$, NS).

\section{Discussion}

This work was aimed to address an essential issue in glucose sensing, the specificity of the sensor's response to glucose. Although the presence of an enzyme should theoretically guarantee this specificity, interference by endogenous or exogenous substances oxidizable at the working electrode applied potential must be considered. Interference by acetaminophen, a commonly used analgesic drug, represents an unresolved issue. There are three main approaches to interference elimi- 
nation: 1) working at a lower potential. This may be possible if a mediator is used to act as a shuttle to transport the electrons produced during the enzymatic oxidation of glucose to the working electrode and if the mediator does not electrocatalytically interact. 2) Introducing an additional membrane capable of screening off this interferent molecule. 3) Use of an enzyme (horseradish peroxidase) to oxidize incoming acetaminophen [12]. A detailed investigation of possible passive membranes suitable for this purpose has been carried out in our laboratories. A Nafion composite membrane was thought to be the most promising design. The aim of the work presented herein was to evaluate, in vitro, and in vivo in rats and human volunteers, the ability of this membrane to reduce acetaminophen interference.

Sensitivity to glucose was not significantly different in vitro for the two groups of sensors, prepared with, or without Nafion. By contrast, the sensitivity to acetaminophen was markedly reduced. Thus, the bias which would be introduced by a given concentration of acetaminophen, estimated by dividing the current produced by this concentration by the sensitivity to glucose, was found to be significantly reduced by the presence of the Nafion membrane. Another important characteristic of the response of a sensor is the response time, which can be defined as the time required to reach $90 \%$ of the maximal current after a stepwise increase in the analyte concentration. Response time to glucose was less than $5 \mathrm{~min}$. By contrast, the increase in the current generated in response to a stepwise increase in acetaminophen concentration was much more sluggish (response time longer than $30 \mathrm{~min}$ ). It is important to point out that this effect was observed both with the non-Nafion and the Nafion sensors; however, the response time was significantly longer for the Nafion sensor. Therefore, the fact that the Nafion membrane reduced the bias introduced by acetaminophen on glucose estimation can be explained both by a reduction in sensitivity to acetaminophen and by an increase in the response time to the analyte.

Next, the two types of sensors were evaluated under in vivo conditions by implantation in the same rat. As expected, the in vivo sensitivity to glucose of both kinds of sensors was much reduced as compared to the sensitivity to glucose determined in vitro, a phenomenon consistently observed with this kind of sensor [13-16]. The acetaminophen infusion technique was designed to produce a rapidly stable plateau around $140 \mu \mathrm{mol} / 1$. The increase in the current produced by both kinds of sensors implanted in the same rat was much reduced for the Nafion sensors.

Acetaminophen interference was next addressed in human volunteers under conditions closer to those of clinical use of the drug (500 mg intake). Under such conditions, the increase in acetaminophen concentration in plasma is transient, with a peak at $60 \mathrm{~min}$ following acetaminophen ingestion, and a subsequent de- crease of $50 \%$ within $2 \mathrm{~h}$. Plasma acetaminophen increased within $1 \mathrm{~h}$ to a $35 \mu \mathrm{mol} / \mathrm{l}$ concentration peak. No change in the current was observed, which is consistent with three phenomena: 1) the pharmacokinetics of acetaminophen transfer from blood to subcutaneous tissue, which was not assessed experimentally in this study, but which is known to be such that during a transient peak in plasma acetaminophen concentration, the acetaminophen level in subcutaneous tissue increases very slowly to reach a much lower peak than that in plasma (Zhang Y, unpublished data);2) the low lump sensitivity to acetaminophen of these sensors, which was defined above; 3 ) the slow response time of the sensor to acetaminophen, which can be demonstrated in vitro (see above).

During the subsequent OGTT, a significant increase in the current was observed during the modest increase, from 4 to $8 \mathrm{mmol} / \mathrm{l}$, in glucose concentration. The calibration of the sensor was performed on the basis of the currents and plasma glucose concentrations observed in time 60 and $120 \mathrm{~min}$ of the trial, i.e. when the plasma acetaminophen concentration was fluctuating. If these fluctuations had had an effect on the current produced by the sensor, one would have expected that the result of the calibration would yield a misestimation of the plasma glucose concentration. Data presented herein demonstrate clearly that the presence of fluctuations in acetaminophen concentration does not jeopardize the ability of the glucose sensor to yield an accurate estimation of plasma glucose concentration, which is the aim of a glucose monitoring system. Furthermore, there was no statistical difference between the estimates of subcutaneous glucose concentration and the concomitant plasma glucose level at the end of the OGTT.

In this human trial, we did not assess the current generated in response to $500 \mathrm{mg}$ acetaminophen ingestion by non-Nafion sensors. However, assuming that the difference in sensitivity observed in rats between both kinds of sensors would be identical in human subcutaneous tissue, one can calculate that a $35 \mu \mathrm{mol} / \mathrm{l}$ acetaminophen concentration should yield a bias of approximately 0.3 and $1.1 \mathrm{mmol} / \mathrm{l}$ for the Nafion, and non-Nafion sensors, respectively. The latter cannot be ignored for instance in this domain of blood glucose concentration where the accuracy of glucose estimation must be optimal (for instance between 3 and $5 \mathrm{mmol} / \mathrm{l}$ for the detection of hypoglycaemia).

The absence of in vivo response to acetaminophen is the result of several mutually interacting factors. As demonstrated, the static sensitivity (response when the sensor achieves a constant output for a constant concentration of acetaminophen) is indeed reduced in those sensors containing the composite Nafion membrane. Equally important is an increase in the response time of the sensor to acetaminophen. If these two properties are combined with the relatively rapidly varying concentration characteristic of acetaminophen 
pharmacokinetics, the resulting "dynamic response" of the sensor should virtually eliminate interference with glucose measurements. Thus, static in vitro experiments and the experiments in rats where acetaminophen infusion in employed correspond to the "worst case" estimation of in vivo sensor performance. Such measurements are nonetheless useful in sensor design and in predicting possible interference.

In conclusion, results presented herein indicate that, 1) the introduction of a Nafion membrane in the design of the sensor significantly reduces the sensitivity to acetaminophen without altering the sensitivity to glucose: how the composite Nafion cellulose acetate present in this sensor reduces the interference by acetaminophen is unclear. Such an interference was present in a sensor using a Nafion membrane as the outer sensor coating [17]. 2) The response time in vitro to acetaminophen is much slower than that to glucose; 3 ) a transient increase of $30-40 \mu \mathrm{mol} / \mathrm{l}$ in plasma acetaminophen concentration, observed after the intake of $500 \mathrm{mg}$ acetaminophen in healthy human subjects, does not produce a significant interfering current. Whether a higher intake can have an effect remains to be determined. However, this paper provides a method for reducing this interference and, in any case for assessing the magnitude of the protective effect of the composite Nafion membrane.

Acknowledgements. This study was supported by Institut National de la Santé et de la Recherche Médicale (grant CNAMTS), National Institute of Health (USA, grant $\mathrm{n}^{\circ}$ DK 30718), Aide aux Jeunes Diabétiques (grant to VP) and Hoechst AG, Frankfurt. We wish to thank Professeur D. Thévenot for constructive criticism of the manuscript.

\section{References}

1. Wilson GS, Thévenot DR (1980) Unmediated amperometric enzyme electrodes. In: Cass AEG (ed) Biosensors: a practical approach. IRL Press, Oxford University Press, Oxford

2. Yamasaki Y, Ueda N, Nao K et al. (1989) Direct measurements of whole blood glucose by a needle-type glucose sensor. Clin Chim Acta 93:93-98

3. Amine K, Kauffman JM, Patriarche GJ, Guilbault GG (1989) Electrochemical behaviour of a lipid modified enzyme electrode. Anal Lett 22 (11 \& 12): 2403-2411
4. Moatti-Sirat D, Velho G, Reach G (1991) Evaluating in vitro and in vito the interference of ascorbate and acetaminophen on glucose detection by a needle-type glucose sensor. Biosens Bioelectron 7: 345-352

5. Saeger S, Preidel W, Lucadou IV, Luprecht L, Lager W (1991) Influence of urea on the glucose measurement by electrocatalytic sensor in the extracorporeal blood circulation of a sheep. Biomed Biochim Acta 50; 7: 885-891

6. Saeger S, Preidel W, Luprecht L (1992) Influence of paracetamol, sulfanilamide and ascorbic acid on the electrocatalytic glucose sensor. Horm Metab Res 24: 504-507

7. Bindra DS, Zhang Y, Wilson GS et al. (1991) Design and in vitro studies of a needle type glucose sensor for subcutaneous monitoring. Anal Chem 63: 1692-1696

8. Sternberg R, Barrau MB, Gangiotti L et al. (1988) Study and development of needle-type multilayer enzyme-based glucose microsensor. Biosensors 4: 27-40

9. Forrest JAH, Clements JA, Prescott LF (1982) Pharmacokinetics of paracetamol. Clin Pharmacokin 7:93-107

10. Gwlit JR, Robertson A, Goldman L, Blanchard AW (1963) The absorption characteristics of paracetamol tablets in man. J Pharm Pharmacol 15: 445-453

11. Velho G, Froguel Ph, Thévenot DR, Reach G (1989) Strategies for calibrating a subcutaneous glucose sensor. Biochem Biomed Acta 48: 957-964

12. Maidan R, Heller A (1991) Elimination of electrooxidizable interferants in glucose electrodes. J Am Chem Soc 113: 90049006

13. Moatti-Sirat D, Capron F, Poitout V et al. (1992) Towards continuous glucose monitoring: in vivo evaluation of a miniaturized glucose sensor implanted for several days in rat subcutaneous tissue. Diabetologia 35: 224-230

14. Rebrin K, Fischer U, Hahn von Dorsche $H$, von Woetke $T$, Abel P, Brunstein E (1992) Subcutaneous glucose monitoring by means of electrochemical sensors: fiction or reality? $\mathrm{J}$ Biomed Eng 14:33-40

15. Poitout V, Moatti-Sirat D, Reach G (1992) Calibration in dogs of a subcutaneous miniaturized glucose sensor using a glucose meter for blood glucose determination. Biosens Bioelectron 7: 587-592

16. Poitout V, Moatti-Sirat D, Reach G et al. (1993) A glucose monitoring system for on line estimation in man of blood glucose concentration using a miniaturized glucose sensor implanted in the subcutaneous tissue, and a wearable control unit. Diabetologia 36: 658-663

17. Moussy F, Harrison DJ, O'Brien DW, Rajotte RV (1993) Peformance of subcutaneously implanted needle-type glucose sensors employing a novel trilayer coating. Anal Chem 65: 2072-2077 